\title{
Development of RNA Targeted Probes for Fluorescence and Electron Microscopy
}

\author{
Philip J. Santangelo
}

Wallace H. Coulter Department of Biomedical Engineering, Georgia Institute of Technology and Emory University, Atlanta, GA 30332

Over the last decade, there has been increasing evidence to suggest that RNA molecules have a wide range of functions in living cells, from physically conveying and interpreting genetic information, to essential catalytic roles, to providing structural support for molecular machines, to gene silencing. These functions are realized through control of their expression level, turnover rates, and through their spatial distribution. In vitro methods that use purified RNA obtained from cell lysates can provide a measure of the RNA expression level within a cell population, but cannot reveal the spatial and temporal variation of RNA. The ability to image specific RNAs in living cells in real-time, as well as in fixed cells with nanometer resolution, promises to provide information on RNA synthesis, processing, transport, and localization. This information should offer new opportunities for advancement in molecular biology, disease pathogenesis, drug discovery, and medical diagnostics. To date, two approaches have been used to image RNA in live cells: (1) fluorescent protein-RNA binding protein (or peptide) fusions are expressed and bind to RNA sequences that have been engineered into the RNA target or the fusion probes themselves have been engineered to bind to native sequences [1-3] and (2) the use of fluorescently-labeled antisense nucleic acids that bind to RNA targets, plasmid-derived or native, via Watson-Crick pairing [4-9].

In this tutorial, the second approach will be explored including a discussion of probe delivery methods for live cells, maximizing sensitivity in both live and fixed cell fluorescence applications, imaging of RNA dynamics using fluorescence, and compatibility with electron microscopy. Examples will include applications of single RNA sensitive, multiply-labeled tetravalent imaging probes (MTRIPs) (Fig. 1), developed in my lab, to the imaging of native $\beta$-actin mRNA dynamics, and the imaging of the viral genomic RNA of two viruses, human Respiratory Syncytial Virus (Fig. 2) and Influenza.

Below are bullet points outlining the major topics to be covered:

- Why understand RNA spatial biology?

- Current approaches for imaging native RNA in live cells, fixed cells and fixed tissue

- Discussion of spatial and temporal scales that need to be addressed with these agents

- Maximizing probe sensitivity for dynamic fluorescence measurements

- Multimodality RNA probes compatible with fluorescence and electron microscopy

References

[1] Fusco, D., et al., Single mRNA molecules demonstrate probabilistic movement in living mammalian cells. Curr Biol, 2003. 13(2): p. 161-7.

[2] Daigle, N. and J. Ellenberg, LambdaN-GFP: an RNA reporter system for live-cell imaging. Nat Methods, 2007. 4(8): p. 633-6. 
[3] Ozawa, T., et al., Imaging dynamics of endogenous mitochondrial RNA in single living cells. Nat Methods, 2007. 4(5): p. 413-9.

[4] Leonetti, J.P., et al., Intracellular distribution of microinjected antisense oligonucleotides. Proc Natl Acad Sci U S A, 1991. 88(7): p. 2702-6.

[5] Politz, J.C., K.L. Taneja, and R.H. Singer, Characterization of hybridization between synthetic oligodeoxynucleotides and RNA in living cells. Nucleic Acids Res, 1995. 23(24): p. 4946-53.

[6] Femino, A.M., et al., Visualization of single RNA transcripts in situ. Science, 1998. 280(5363): p. 585-90.

[7] Raj, A., et al., Imaging individual mRNA molecules using multiple singly labeled probes. Nat Methods, 2008. 5(10): p. 877-9.

[8] Bratu, D.P., et al., Visualizing the distribution and transport of mRNAs in living cells. Proc Natl Acad Sci U S A, 2003. 100(23): p. 13308-13.

[9] Santangelo, P.J., et al., Single molecule-sensitive probes for imaging RNA in live cells. Nat Methods, 2009. 6(5): p. 347-9.
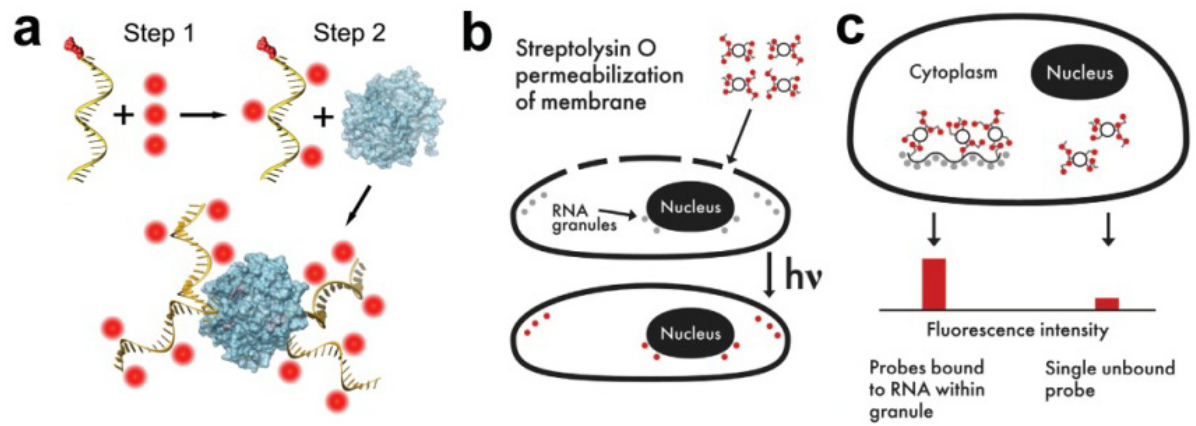

FIG. 1. MTRIPs, imaging strategy, and evaluation of probe delivery via streptolysin O within a live cell. (a) MTRIPs; biotinylated, fluorophore-conjugated chimera oligonucleotides bound to streptavidin. (b) Cells incubated with SLO/medium/probe are permeabilized by SLO allowing probe diffusion through the pores and rapid binding to target. (c) When multiple probes bind to a single RNA, the signal-to-background ratio is enhanced and allows for target recognition.

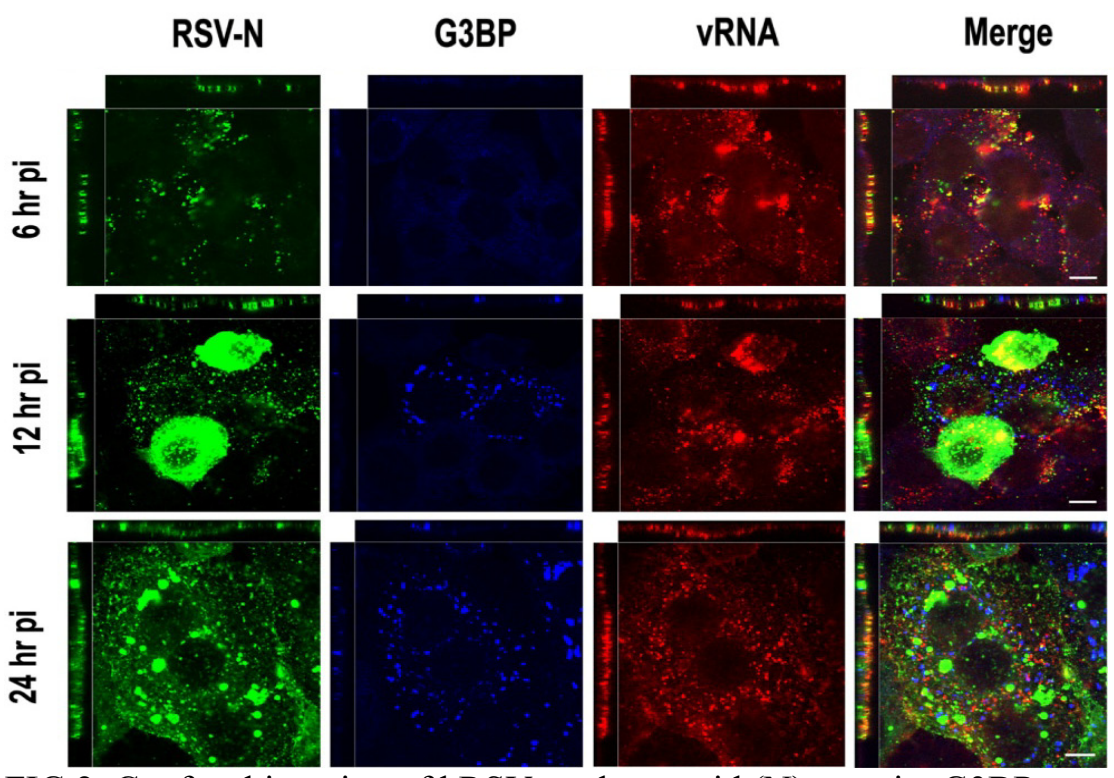

FIG 2. Confocal imaging of hRSV nucleocapsid (N) protein, G3BP, a stress granule marker, and the genomic vRNA via MTRIPs in HEp-2 cells. Scale bar represents $10 \mu \mathrm{m}$. 